\title{
Refractory Nonconvulsive Status Epilepticus with Favorable Outcome in a Patient with Marchiafava-Bignami Disease
}

\author{
Ryul Kim ${ }^{a}$ \\ Hee-Jin Chob \\ Ho-Won Lee b,c $^{\text {bo }}$ \\ Jin-Sun Jun ${ }^{\mathrm{a}, \mathrm{d}}$ \\ aDepartments of Neurology and \\ dNeurosurgery, \\ Seoul National University Hospital, \\ Seoul, Korea \\ ${ }^{b}$ Department of Neurology, \\ School of Medicine, \\ Kyungpook National University, \\ Kyungpook National University \\ Chilgok Hospital, Daegu, Korea \\ 'Brain Science and Engineering Institute, \\ Kyungpook National University, Daegu, \\ Korea
}

Dear Editor,

Marchiafava-Bignami disease (MBD) is a rare condition mainly associated with chronic alcoholism that is characterized by demyelination and necrosis of the corpus callosum. ${ }^{1} \mathrm{MBD}$ produces various neurological symptoms, including an altered mental state, gait difficulty, cognitive dysfunction, and seizure. ${ }^{1}$ Although status epilepticus has rarely been reported in patients with MBD, this condition can lead to poor outcomes. ${ }^{2}$ Here we report a patient showing a favorable outcome after refractory nonconvulsive status epilepticus (NCSE) as an initial manifestation of MBD.

A 58-year-old man presented in an acute confused state with intermittent upward eyeball deviation that had developed a few hours prior to seeking treatment. He had a history of chronic high alcohol intake, consuming about 2 bottles of Korean soju daily. The patient's wife mentioned that he had consumed alcohol within 6 hours before the onset of the symptoms. He had a history of early esophageal cancer, which had been treated with endoscopic submucosal dissection 2 years previously. He had no history of recent medication. A neurological examination revealed that he was disoriented and had a fluctuating level of consciousness. Routine blood tests (including of the thiamine level) and cerebrospinal fluid studies revealed no abnormalities. Brain magnetic resonance imaging (MRI) performed at admission revealed hyperintense lesions involving the splenium and genu of the corpus callosum and the cerebral cortex (Fig. 1A-D), which was consistent with MBD. Electroencephalography (EEG) revealed periodic rhythmic theta-to-delta activity suggestive of NCSE (Supplementary Fig. 1 in the online-only Data Supplement).

He was started on intravenous (IV) thiamine at $200 \mathrm{mg}$ /day, in addition to IV lorazepam $(0.1 \mathrm{mg} / \mathrm{kg}$ ) followed by IV fosphenytoin (30 mg/kg loading dose), but his clinical and electrographic seizures persisted. Seizure control was achieved on day 4 after adding levetirace$\operatorname{tam}(2,000 \mathrm{mg} /$ day $)$ and lacosamide (400 $\mathrm{mg} /$ day). On day 7 he was oriented and was able to walk with some assistance. He was maintained on IV thiamine for 28 days, followed by oral thiamine at $30 \mathrm{mg} /$ day. Follow-up MRI at 1 month after the onset of symptoms revealed persistent hyperintense lesions of the corpus callosum, with some atrophic changes (Fig. 1E-I). At the 2-month follow-up he was able to carry out many of his usual activities without assistance. He did not experience any symptom indicative of a seizure while receiving maintenance levetiracetam ( $300 \mathrm{mg} /$ day) and lacosamide ( $400 \mathrm{mg} /$ day).

This patient represents a unique case of MBD presenting with refractory NCSE. The patient had a normal blood thiamine level, but MBD can be diagnosed regardless of the thiamine level. ${ }^{1}$ To the best of our knowledge NCSE has not been reported previously in a patient with MBD, and so the present findings are the first to suggest that MBD can be involved in the etiology of NCSE.

@ This is an Open Access article distributed under the terms of the Creative Commons Attribution Non-Commercial License (https://creativecommons.org/licenses/by-nc/4.0) which permits unrestricted non-commercial use, distribution, and reproduction in any medium, provided the original work is properly cited. 

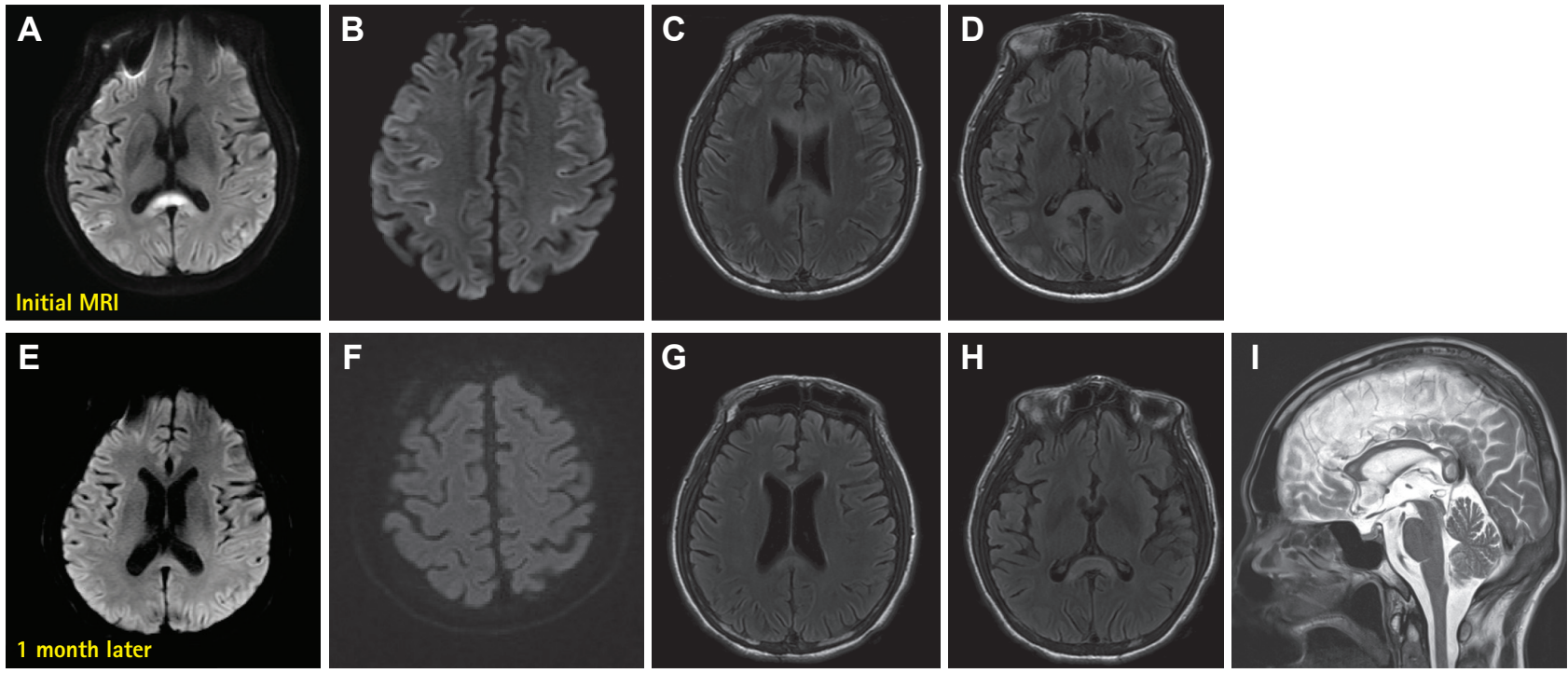

Fig. 1. MRI findings. The initial MRI revealed increased signal intensities in the splenium and genu of the corpus callosum and the cerebral cortex in DWI ( $A$ and B) and axial FLAIR imaging (C and D). Follow-up MRI performed 1 month later revealed complete resolution of the abnormal DWI signals ( $E$ and F), but persistent lesion hyperintensities with some atrophic changes were observed on axial FLAIR images $(G$ and $H$ ) and sagittal T2-weighted images (I). DWI: diffusion-weighted imaging, FLAIR: fluid-attenuated inversion recovery, MRI: magnetic resonance imaging.

Other alcohol-related conditions such as alcohol-withdrawal seizures and subacute encephalopathy with seizures in alcoholics (SESA syndrome) should have been considered in this case, but some of the findings were more strongly suggestive of the seizures being due to MBD. Alcohol-withdrawal seizures generally occur $12-48$ hours after the abrupt cessation of alcohol intake in alcohol-dependent patients. ${ }^{3}$ However, our patient had consumed alcohol during the 6-hour period before the onset of symptoms. Furthermore, alcoholwithdrawal seizures typically manifest with generalized tonic-clonic seizures or (less frequently) partial seizures over a short duration, ${ }^{4}$ whereas our patient experienced prolonged nonconvulsive seizures. On the other hand, SESA syndrome usually shows periodic lateralized epileptiform discharges and/ or focal slow delta activity on EEG. The MRI characteristics of SESA syndrome generally include multi-infarct changes with predominance in the subcortical and periventricular areas, ${ }^{5}$ but there were no such findings in our patient.

There are two clinicoradiological subtypes of MBD. ${ }^{6}$ Type A shows major impairment of consciousness, the involvement of the entire corpus callosum, and poor outcomes, whereas type $B$ is characterized by slight impairment of consciousness, partial involvement of the corpus callosum, and favorable outcomes. Extracallosal lesions are present more frequently in MBD of type A than of type B. The clinical and imaging features in our patient were compatible with type $\mathrm{A}$, but he showed a relatively good prognosis despite the occurrence of refractory NCSE. This finding may emphasize the importance of prompt therapy with parenteral thiamine. Hillbom et al. ${ }^{1}$ showed that a favorable outcome was associated with initiating thiamine within 2 weeks after the onset of symptoms, while we administered thiamine within 1 day after the onset of symptoms.

\section{Supplementary Materials}

The online-only Data Supplement is available with this article at https://doi.org/10.3988/jen.2019.15.3.393.

\section{Conflicts of Interest}

The authors have no financial conflicts of interest.

\section{REFERENCES}

1. Hillbom M, Saloheimo P, Fujioka S, Wszolek ZK, Juvela S, Leone MA. Diagnosis and management of Marchiafava-Bignami disease: a review of CT/MRI confirmed cases. J Neurol Neurosurg Psychiatry 2014;85:168-173.

2. Navarro JF, Noriega S. Marchiafava-Bignami disease. Rev Neurol 1999; 28:519-523.

3. O'Connor CM. Alcohol abuse and dependence (chap 32). In: Goldman L, Schafer AI, editors. Goldman-Cecil Medicine. 24th ed. Philadelphia: Elsevier Saunders, 2012.

4. Rogawski MA. Update on the neurobiology of alcohol withdrawal seizures. Epilepsy Curr 2005;5:225-230.

5. Fernández-Torre JL, Kaplan PW. Subacute encephalopathy with seizures in alcoholics (SESA syndrome) revisited. Seizure 2014;23:393396.

6. Heinrich A, Runge U, Khaw AV. Clinicoradiologic subtypes of Marchiafava-Bignami disease. J Neurol 2004;251:1050-1059. 